\title{
An unusual phase transition driven by vibrational entropy changes in a hybrid organic-inorganic perovskite
}

\author{
Wenjuan Wei, Wei Li, Keith T. Butler, Guoqiang Feng, \\ Christopher J. Howard, Michael A. Carpenter, Peixiang Lu, \\ Aron Walsh and Anthony K. Cheetham
}

\section{Published version information}

This is the peer reviewed version of the following article:

Citation: W Wei et al. "An unusual phase transition driven by vibrational entropy changes in a hybrid organic-inorganic perovskite."Angewandte Chemie International Edition, vol. 57, no. 29 (2018): 8932-8936.

DOI: $10.1002 / a n i e .201803176$

Which has been published in final form at DOI above. This article may be used for non-commercial purposes in accordance with Wiley-VCH terms and conditions for self-archiving.

Please cite only the published version using the reference above. This is the citation assigned by the publisher at the time of issuing the AAM. Please check the publisher's website for any updates. 


\title{
An Unusual Vibrational Entropy Driven Phase Transition in a
}

\section{Hybrid Organic-Inorganic Perovskite}

\author{
Wenjuan Wei ${ }^{1}$, Wei Li $^{1 *}$, Keith T. Butler ${ }^{2 *}$, Guoqiang Feng ${ }^{1}$, Christopher J. Howard ${ }^{3}$, Song \\ $\mathrm{Gao}^{4 *}$, Michael A. Carpenter ${ }^{*}$, Peixiang Lu ${ }^{1}$ and Aron Walsh ${ }^{6}$, Anthony K. Cheetham ${ }^{7}$ \\ ${ }^{1}$ Department of Physics, Huazhong University of Science \& Technology, Luoyu Road, Wuhan, China \\ ${ }^{2}$ ISIS Neutron and Muon Source, Rutherford Appleton Laboratory, Didcot, OX110DQ \\ ${ }^{3}$ School of Engineering, University of Newcastle, New South Wales 2308, Australia \\ ${ }^{4}$ College of Chemistry and Molecular Engineering, Peking University, Beijing 100871, China \\ ${ }^{5}$ Department of Earth Sciences, University of Cambridge, Downing Street, Cambridge, CB2 3EQ, UK \\ ${ }^{6}$ Department of Materials, Imperial College London, Royal School of Mines, Exhibition Road, London SW7 \\ $2 A Z, U K$
}

Phase transitions (PTs) of $\mathrm{ABX}_{3}$ hybrid organic-inorganic perovskites (HOIPs) can endow many important electrical, magnetic and optical properties which are the key for their applicable functionalities. In this regard, elucidating their mechanisms has become an essential forefront in the field. Hitherto, the driving forces of their PTs were largely limited to the $\mathrm{BX}_{6}$ octahedral tilting (displacive transitions) and molecular ordering of the A-site (order-disorder transitions), while other atomic factors have attracted little attention. Here, we uncover a complex ferroelastic PT in a HOIP, $\left[\mathrm{CH}_{3} \mathrm{NH}_{3}\right]\left[\mathrm{Mn}_{(}\left(\mathrm{N}_{3}\right)_{3}\right]$, based on structural characterisations and $a b$ initio lattice dynamics calculations. This unusual first-order PT, between two ordered phases, is neither displacive nor orderdisorder, but primarily driven by collective atomic vibrations of the whole lattice, along with concurrent molecular displacement and peculiar octahedral tilting. Moreover, the atomic vibrational gain is the origin of the significant entropy change between the low and high temperature structures, and plays a critical role in driving the transition. This finding offers an alternative pathway for designing new ferroic PTs and related physical properties in HOIPs and other hybrid crystals.

*e-mail: w1276@hust.edu.cn; k.t.bulter@bath.ac.uk 


\section{gaosong@pku.edu.cn; mc43@esc.cam.ac.uk}

Hybrid organic-inorganic perovskites (HOIPs) have attracted growing interest in the past several years due to their potential applications in the fields of photovoltaics, optoelectronics and sensing devices ${ }^{1}$. Similar to their conventional oxide counterparts, these HOIPs exhibit abundant structural transitions in response to external perturbation such as temperature or pressure $^{2-3}$. Since these phase transitions (PTs) can often endow significant changes in the electrical, magnetic and optical properties, which are of vital importance for the design and fabrication of functional devices, it is crucial to fully understand their origins from an atomic scale. For example, it has been found that the first-order tetragonal-to-orthorhombic transition of $\mathrm{MAPbI}_{3}(\mathrm{MA}=$ methylammonium $)$ below $160 \mathrm{~K}-$ driven by the soft modes of $\mathrm{PbI}_{6}$ octahedral tilting and the MA cation ordering - could have a significant impact on its electronphonon coupling and corresponding photovoltaic properties ${ }^{4}$.

In traditional inorganic perovskites, the $\mathrm{A}$-site displacement and $\mathrm{BX}_{6}$ octahedral tilting are the primary driving forces of $\mathrm{PTs}^{5-6}$, these are displacive transitions. However, the presence of organic species on the A- and/or X-sites in HOIPs significantly complicates the symmetry breaking process ${ }^{2,7}$. Though octahedral tilting patterns in HOIPs are generally reminiscent of those in conventional perovskites, these much larger and longer X-sites offer additional freedom for the octahedra to distort, hence offering new tilting pathways. The recent discovery that adjacent octahedra in $[\mathrm{TMA}]\left[\mathrm{Mn}\left(\mathrm{N}_{3}\right)_{3}\right](\mathrm{TMA}=$ tetramethylammonium) and other azide HOIPs tilt in the same sense indeed proves such new possibilities ${ }^{8-10}$. On the other hand, the dynamic movement of the organic A-site from one state to another, which normally appears as order-disorder switching via rotational and configurational changes, can also induce symmetry changes $^{11-14}$. The accompanying alterations of hydrogen bonding ${ }^{15}$ and dispersive forces ${ }^{16}$ during the dynamic switching will in turn influence the PT. Such complex synergies in the A- 
site have been found to be the main origin of ferroelectricity in some HOIPs (e.g. $[\mathrm{DMA}]\left[\mathrm{Co}(\mathrm{DCOO})_{3}\right], \mathrm{DMA}=$ dimethylammonium $)^{17}$, in stark contrast to the displacive nature of electrical ordering in perovskite oxides ${ }^{18-19}$.

Although the importance of PTs of HOIPs has attracted increasing awareness, most studies were focused on the aforementioned driving factors of molecular ordering and displacement ${ }^{2}$. As one prominent difference between HOIPs and inorganic perovskites is their atomic weight, the presence of substantially lighter elements such as hydrogen and carbon could lead to remarkable energetic changes across the transition if their vibrational entropic effects are significant enough. Such vibrational entropy effects have been found to be the primary factor for driving the polymorphism in hybrid formates by recent lattice dynamics calculations ${ }^{20}$. Here we report on an unusual vibrational entropy driven PT in an azide HOIP, $[\mathrm{MA}]\left[\mathrm{Mn}\left(\mathrm{N}_{3}\right)_{3}\right]$ on the basis of comprehensive structural and thermodynamic characterisations, as well as extensive lattice dynamics calculations. We show that the complex first-order PT in $[\mathrm{MA}]\left[\mathrm{Mn}\left(\mathrm{N}_{3}\right)_{3}\right]$ involves the synergistic motions of the octahedral tilting, rotation and displacement of the MA cation, and motion of the azide group. More importantly, we reveal that the significant entropy change across the transition arises from the vibrational effects of all atoms which in return are the major source for thermally driving the PT.

$[\mathrm{MA}]\left[\mathrm{Mn}\left(\mathrm{N}_{3}\right)_{3}\right]$ has an $\mathrm{ABX}_{3}$ perovskite structure, in which the A-, B- and $\mathrm{X}$-sites are $\mathrm{MA}^{+}$, $\mathrm{Mn}^{2+}$ and azide groups, respectively. The high temperature (HT) structure (270 K, Fig. 1a) of [MA] $\left[\mathrm{Mn}\left(\mathrm{N}_{3}\right)_{3}\right]$ crystallizes in the monoclinic space group $P 2_{1} / c$, with $a=9.0311(10), b=$ 7.3935(8), $c=12.5463(13) \AA, \beta=102.434(11)^{\circ}$ and $V=818.09(16) \AA^{3}$, in which the fully ordered $\mathrm{MA}^{+}$is hydrogen-bonded to the $\left[\mathrm{Mn}\left(\mathrm{N}_{3}\right)_{3}\right]^{-}$perovskite framework ${ }^{21}$. There are four hydrogen bonds in each pseudo-perovskite unit and the $\mathrm{N} \cdots \mathrm{N}$ distances and $\mathrm{N}-\mathrm{H} \cdots \mathrm{N}$ angles 
are 2.967(3)-3.152(4) $\AA$ and $130.0(5)-171.4(4)^{\circ}$, respectively. Notably, the MA cation is located in the perovskite cavity at an off-center position along the $c$-axis and the adjacent $\mathrm{MnN}_{6}$ octahedra distort along this direction. According to the Glazer notation, the octahedral tilting system of the HT phase would be $a^{-} b^{-} b^{-22}$. Referred to a notional perovskite-like cubic parent structure with space group $P m-3 m$ and cell parameter $a_{c}$, this the HT $P 2_{1} / c$ structure has cell dimensions $\sim \sqrt{2} a_{\mathrm{c}} * \sqrt{2} a_{\mathrm{c}} * 2 a_{\mathrm{c}}$
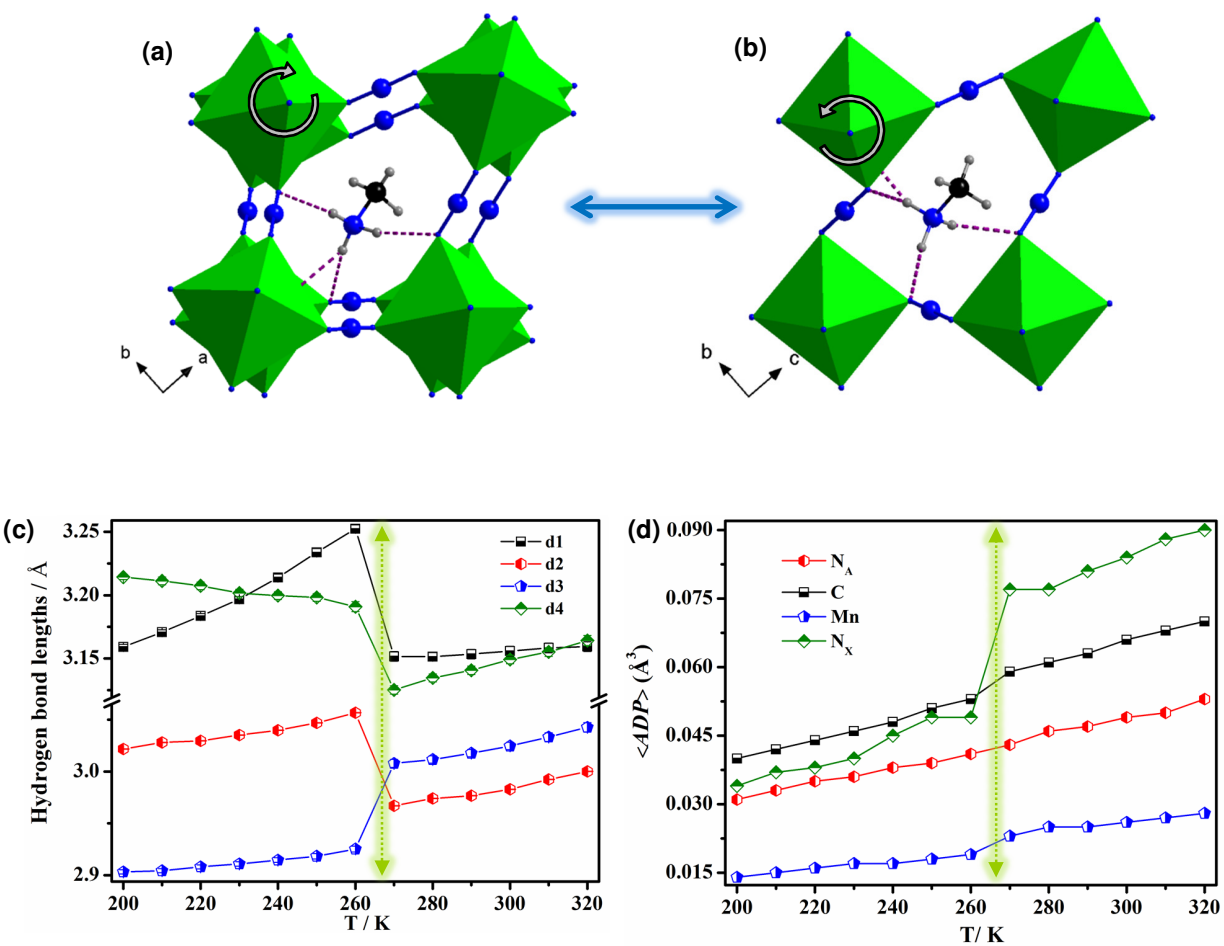

Figure 1. (a) The crystal structure of HT $(270 \mathrm{~K})$ viewed down the $c$ axis. (b) The crystal structure of LT $(260 \mathrm{~K})$ viewed along the $a$ axis. Violet dotted lines signify the hydrogen bonds. The grey arrows indicate the rotation directions of octahedra during the PT. Colour scheme: Mn, emerald; C, black; N, blue; H, 25\% grey. (c) Hydrogen bond lengths as a function of temperature from 200 to $320 \mathrm{~K}$. (d) Temperature dependencies of atomic displacement parameters (ADPs) of Mn, $\mathrm{C}, \mathrm{N}_{\mathrm{A}}$ and $\mathrm{N}_{\mathrm{X}}$ atoms. Note: $\mathrm{N}_{\mathrm{A}}$ and $\mathrm{N}_{\mathrm{X}}$ represent the nitrogen atoms of the A- and X-sites, respectively; the emerald dashed line separates the LT and HT phase regions. 
Upon cooling, $[\mathrm{MA}]\left[\mathrm{Mn}\left(\mathrm{N}_{3}\right)_{3}\right]$ undergoes a PT at about $264 \mathrm{~K}$ to a low temperature phase (LT) with the same monoclinic space group $P 2_{1} / c$, but with $a=6.2218(4), b=7.2500(4), c=$ $18.3398(11) \AA, \beta=102.075(5)^{\circ}$ and $V=808.97(8) \AA^{3}$ (Fig. 1b) ${ }^{21}$. With respect to the same parent cubic structure as referred to above, the cell dimensions of the LT structure are $\sim a_{\mathrm{c}}{ }^{*}$ $\sqrt{2} a_{\mathrm{c}} * 2 \sqrt{2} a_{\mathrm{c}}$. This structure does not have a group-subgroup relationship with the HT structure so the transition must be first order in character, with a complex pattern of changes which involves all the A-, B- and X-sites. Specifically, the MA cation exhibits both displacement and rotation about the $\mathrm{C}-\mathrm{N}$ axis, and the $\mathrm{MnN}_{6}$ octahedral tilts change dramatically (the Glazer notation is no longer applicable) along with significant movement of the azide groups. This is accompanied by the breaking and reforming of hydrogen bonding with $\mathrm{N} \cdots \mathrm{N}$ distances of 2.925(2)-3.252(3) $\AA$ and $\mathrm{N}-\mathrm{H} \cdots \mathrm{N}$ angles of 149.2(3)-158.6(3) ${ }^{\circ}$ (Fig. 1b). Strikingly, three newly constructed hydrogen bonds in the LT structure are about $2.1-3.2 \%$ longer than those in the HT structure though the fourth one is slightly shorter (2.8\%) (Fig. 1c). This results in overall weakening of the hydrogen-bonding when crossing the transition from HT to LT, which has not been reported in any other HOIPs ${ }^{2}$. However, the thermal vibrations of all atoms in $[\mathrm{MA}]\left[\mathrm{Mn}\left(\mathrm{N}_{3}\right)_{3}\right]$ show a normal trend, where the atomic displacement parameters (ADPs) of C, $\mathrm{N}_{\mathrm{A}}, \mathrm{Mn}$ and $\mathrm{N}_{\mathrm{X}}$ all decrease upon cooling (Fig. 1d). Additional solid state ${ }^{1} \mathrm{H}$ magic-angle spinning nuclear magnetic resonance (MAS NMR) measurements reveal the thermal vibration of hydrogen atoms in the perovskite structure. As seen from Fig. S3, the chemical shift of hydrogen atoms on the $-\mathrm{NH}_{3}$ group reduces from 6.6 to $6.3 \mathrm{ppm}$, with decreasing intensity, when cooling from 323 to $223 \mathrm{~K}$. This indicates increasing shielding effects of the nitrogen atom with respect to the attached hydrogens and, hence, weakening the vibration of amino hydrogens upon cooling. Such an unusual phenomenon arises from the competing effects of electrostatic interactions and hydrogen bonding in the perovskite unit across the transition. The unit cell volume contracts by about $1.13 \%$ when cooling from 270 to $260 \mathrm{~K}$, leading to a 
significant increase of electrostatic interactions between the MA cation and the perovskite host, which compensate for the lengthening of the hydrogen bonding and result in decreasing atomic displacement parameters (ADPs) from HT to LT. Another interesting feature of the transition is that the azide group exhibits both cis-EE and trans-EE coordination modes rather than pure cis-EE in the HT phase ${ }^{21}$.

DSC experiments provide thermodynamic information about the transition (Fig. S2a). The temperature dependencies of heat flow show an anomaly at $277 \mathrm{~K}$ on heating and at $264 \mathrm{~K}$ on cooling, in agreement with the literature ${ }^{21}$. The width of the thermal hysteresis of $13 \mathrm{~K}$ and sharp peaks observed at both heating and cooling confirm the strong first-order nature of the transition. Temperature dependencies of entropy $\left(S_{\text {Exp }}\right)$, shown in Fig. S2b, demonstrate that the associated entropy change $\left(\Delta S_{\mathrm{Exp}}\right)$ is approximately $4.35 \mathrm{~J} \mathrm{~K}^{-1} \mathrm{~mol}^{-1}$. This large value is close to that of a two-fold order-disorder transition $\left(\Delta S=R \ln N_{2} / N_{I}=R \ln 2=5.76 \mathrm{~J} \mathrm{~K}^{-1} \mathrm{~mol}^{-1}\right.$, where $N$ represents the ratio of possible configurations after and before PT and $R$ is the gas constant), though our single crystal X-ray diffraction studies unambiguously exclude the existence of any structural disorder in the HT phase. Such an unusually large entropy change suggests that the whole HT structure exhibits a dramatic increase in the vibrational degrees of freedom and corresponding active phonon modes, in comparison with the LT structure.

In order to better understand these structures, the $\left[\mathrm{Mn}\left(\mathrm{N}_{3}\right)_{3}\right]^{-}$frameworks have been compared with the framework of a notional cubic parent framework in space group $P m-3 m$. The computer program ISODISTORT ${ }^{23}$ has been used to identify the most important distortions of these frameworks. A very significant distortion in each structure is associated with irreducible representation (irrep) $M_{3}^{-}$(at $\left.k=1 / 2,1 / 2,0\right)$; this appears to involve the displacements of the $\mathrm{Mn}$ and neighboring $\mathrm{N}$ alternately above and below the average plane. In the HT structure there is 
also significant distortion associated with irrep $R_{4}{ }^{+}$, well known in perovskites to give out-ofphase octahedral tilting and in this case the tilt system $a^{-} b^{-} b^{-}$. In the LT structure, there is no contribution from $R_{4}{ }^{+}$, but rather a significant contribution from irrep $\Sigma_{2}$ (at $\left.k=1 / 4,1 / 4,0\right)$, which gives a pattern of tilting such that pairs of neighbouring octahedra are tilted in the same sense (Fig. S1b). Although other distortions are present, it is sufficient to couple $M_{3}^{-}$with $R_{4}^{+}$, and $M_{3}{ }^{-}$with $\Sigma_{2}$, to give the HT and LT structures respectively\#. From the perspective of the framework, the HT to LT transition may be seen largely as a change from a conventional $a^{-} b^{-}$ $b^{-}$tilt system to the unusual $\Sigma_{2}$ tilt system against the background of notional cubic framework distorted by irrep $\mathrm{M}_{3}{ }^{-}$. Although the changes in the framework may be interesting, it is very likely that these changes are driven by changes in the orientations and locations of the MA entities in the cavities.

Both HT and LT structures are ferroelastic with respect to the parent cubic structure and the HT phase does indeed contain ferroelastic twin walls at room temperature (Fig. S4). Even though there is no group-subgroup relationship between the HT and LT phases, it is likely that the latter will also contain abundant ferroelastic twin walls. The latter may be driven by the locations of the cavity MA entities because $\Sigma_{2}$ at $\boldsymbol{k}=1 / 4,1 / 4,0$ and $M_{3}{ }^{-}$at $\boldsymbol{k}=1 / 2,1 / 2,0$ in the Brillouin zone would give the required $P 2_{1} / c$ space group ${ }^{7}$.

As has been found in many conventional perovskites, any strains coupled to changes in structure associated with a PT necessarily lead to changes in the elastic properties, while acoustic losses provide insights into the dynamics of strain-related microstructures ${ }^{24}$. Resonant ultrasound spectroscopy (RUS) has been used here to probe these aspects of both the HT and

\#It is found very readily using ISOSUBGROUP ${ }^{25}$ that such an analysis gives both structures in space group $P 2_{1} / c$; for the HT structure on a cell $(1,1,0),(-1,1,0),(0,0,2)$ with an origin of $(0,1 / 2,1 / 2)$ with respect to the notional perovskite parent, and for the LT structure on a cell $(0,1,0),(-1,0,1),(2,0,2)$, origin $(0,0,1 / 2)$, as observed. 
LT phases, as well as the transition between them. Selected RUS spectra from a single crystal collected during both heating and cooling sequences between 100 and $280 \mathrm{~K}$ are shown in Fig. S5a. Data extracted from fitting of selected cooling peaks in these are shown in Fig. 2. The square of the peak frequency, $f$, of selected peaks scales with different combinations of the single crystal elastic constants and shows a sharp discontinuity at the transition point. There is no precursor softening or stiffening as the transition is approached from above and the LT phase is elastically stiffer than the HT phase by $\sim 8-12 \%$ (Fig. $2 \mathrm{a}$ ). All the resonance modes show the same pattern, which is probably related simply to the increased density of the LT phase. The trend is reversed on heating.
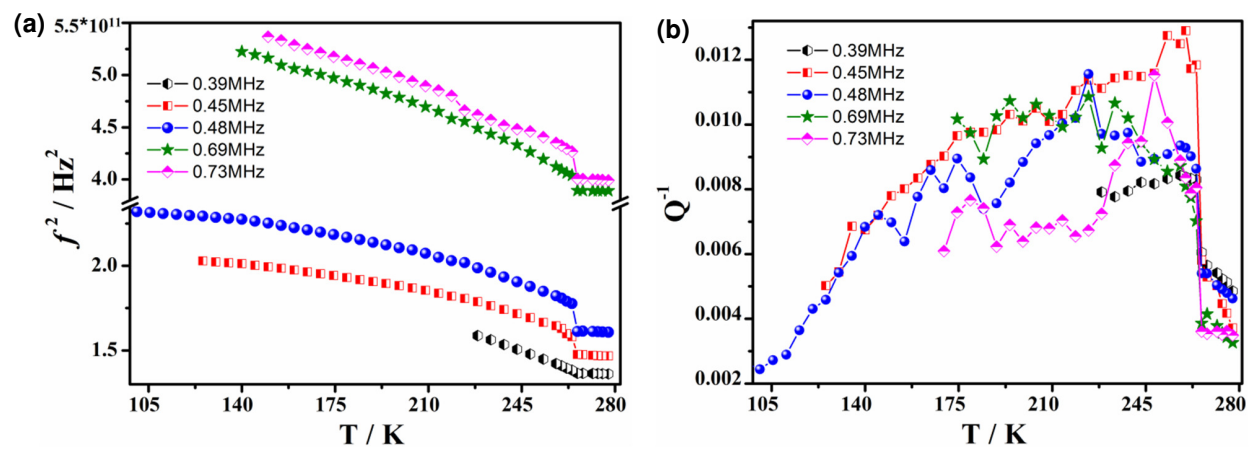

Figure 2. Temperature dependencies of $f^{2}$ (a) and $Q^{-1}(\mathbf{b})$ from fitting of selected cooling resonance peaks of RUS spectra.

The inverse mechanical quality factor, $Q^{-1}$, derived from peak widths (as described in the supplementary information) is a measure of acoustic $\operatorname{loss}^{26}$. It shows a sharp increase between low loss from the HT phase to a wide temperature interval of high loss from the LT phase (Fig. $2 b$ ), with a return to the lowest values below $\sim 100 \mathrm{~K}$. There are three possible causes. The first is simply due to the development of cracks arising from the large volume change on cooling through the transition. Values of $Q^{-1}$ for the HT phase do not return to their initial relatively 
low values after cycling through the transition, suggesting that some micro-cracking might occur, but there is still a significant decrease on reheating through the transition temperature. An extrinsic origin for the increased loss would most likely be from ferroelastic twin walls which are mobile in response to external stress (Fig. S4) ${ }^{24}$, perhaps inherited from the parent HT structure (Fig. S6). However, an intrinsic origin is also possible and could be analogous to the acoustic properties of the mineral lawsonite, $\mathrm{CaAl}_{2} \mathrm{Si}_{2} \mathrm{O}_{7}(\mathrm{OH})_{2} \cdot \mathrm{H}_{2} \mathrm{O}$, in which it appears that relaxations of hydrogen bonding occur in response to the external stress ${ }^{27}$. In $[\mathrm{MA}]\left[\mathrm{Mn}\left(\mathrm{N}_{3}\right)_{3}\right]$, such relaxations develop in RUS measurements at $\sim 0.1-1 \mathrm{MHz}$, implying a time scale of $\sim 10^{-5}-10^{-6} \mathrm{~s}$ for the relaxation process of hydrogen bonding between the MA cation and $\left[\mathrm{Mn}\left(\mathrm{N}_{3}\right)_{3}\right]^{-}$framework. The acoustic loss pattern in $[\mathrm{MA}]\left[\mathrm{Mn}\left(\mathrm{N}_{3}\right)_{3}\right]$ spans $\sim 100 \mathrm{~K}$, which is substantially broader than has been observed in association with a PT driven by the configurational order-disorder of the A-site organic amine cations in $\left[\left(\mathrm{CH}_{2}\right)_{3} \mathrm{NH}\right]\left[\mathrm{Mn}(\mathrm{HCOO})_{3}\right]^{28}$ and $[\mathrm{DMA}]\left[\mathrm{M}(\mathrm{HCOO})_{3}\right](\mathrm{M}=\mathrm{Mn} \text { and } \mathrm{Co})^{29}$, suggesting a fundamentally different origin.

To fully understand the PT mechanism and origin of the associated large entropy change across the PT, we have applied lattice dynamics calculations to probe the driving forces ${ }^{30-31}$. The Gibbs free energy $(G)$ is calculated by applying the harmonic approximation methodology from

$$
G=U+E_{V i b}-T S_{V i b}(1)
$$

where the internal energy of the system $U$ can be obtained from density functional theory (DFT) calculations. The phonon free energy $\left(E_{V i b}+S_{V i b}\right)$ arising from harmonic vibrations of the lattice is calculated using the frozen phonon approximation. The Mn centres were treated in a high spin state and the spin orientations were allowed to relax automatically. At the temperatures of the PT considered there is no magnetic ordering present in the material; the energy differences between different magnetic orderings are also an order of magnitude smaller than either 
differences in internal energy or vibrational entropy, so would have little effect on our analysis. The energy related to the magnetic ordering is treated using ... which is trivial to $G$ and is not considered here (Keith, can you add a bit more information here?). The vibrational entropy $\left(S_{V i b}\right)$ is calculated from the phonon density of states (DOS) of all positive phonon modes according to

$$
S_{V i b}(T)=3 k_{\mathrm{B}} \int_{0}^{\infty} g(\varepsilon)\{[n(\varepsilon)+1] \ln [n(\varepsilon)+1]-n(\varepsilon) \ln [n(\varepsilon)]\} d \varepsilon
$$

where $k_{\mathrm{B}}$ is the Boltzmann constant, $g(\varepsilon)$ is the normalized phonon DOS with energy $\varepsilon=\hbar \omega$ ( $\omega$ is mode frequency), $n(\varepsilon)$ is Bose-Einstein population of a state of energy $\varepsilon$ at temperature $T$.

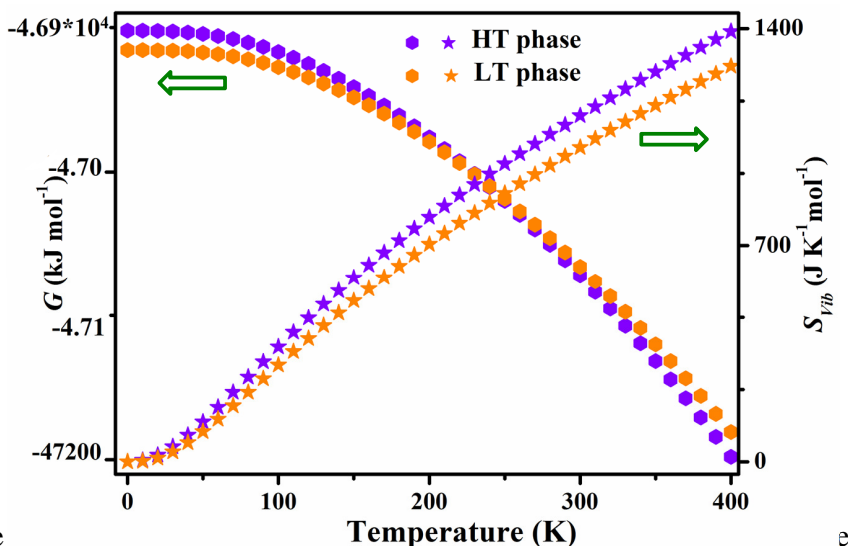

Figure 3. The Temperature (K)

exagons) for the HT and LT phases calculated across a 0 - $400 \mathrm{~K}$ temperature range.

The contributions of the phonon modes to $S_{V i b}$ of these two phases are shown in Fig. S7, which demonstrates that the HT structure exhibits larger vibrational effects at almost all phonon energies. In addition, the left plots in Fig. 3 show $G$ of the HT and LT phases as a function of temperature. The internal energy difference $(\Delta U)$ between the two phases is constant with varying temperature, therefore cannot account for the crossover of the phase stability. Additionally, since both the HT and LT structures have similar thermal expansion as observed 
from VT-SCXRD (Fig. S8), $p V$ does not contribute to the PT significantly. This means that the dominant effect for driving the transition arises from the vibrational entropy term $\left(E_{V i b}-T S_{V i b}\right)$, as deduced from equation 1 . The lack of any difference in configurational entropy across the transition distinguishes this system from the scenario in other HOIPs (e.g. [MA] $\left[\mathrm{PbI}_{3}\right]^{32}$ and $\left.[\mathrm{DMA}]\left[\mathrm{M}(\mathrm{HCOO})_{3}\right](\mathrm{M}=\mathrm{Mn}, \mathrm{Co} \text { and } \mathrm{Ni})^{33}\right)$, where dynamic configurational ordering mainly contributes to the difference in entropy between competing phases. $E_{V i b}$ is greater in the HT phase than the LT phase at all temperatures, and destabilises the HT phase by $3.43 \mathrm{kJmol}^{-1}$ at the phase transition point, therefore the driving force for the HT phase becoming more stable can only come from $S_{V i b}$. Furthermore, the right panel of Fig. 3 shows that the $S_{V i b}$ of the HT phase is greater than that of the LT structure. As the temperature increases so the $T S_{V i b}$ contribution to $G$ becomes more pronounced and eventually the HT phase becomes thermodynamically more favourable than the LT phase at about $220 \mathrm{~K}$, which is in reasonable agreement with the experimental value at around $265 \mathrm{~K}$. At the transition we calculate a value of $\Delta S_{V i b}$ of $22.75 \mathrm{~J} \mathrm{~K}^{-1} \mathrm{~mol}^{-1}$ greater than the measured value $\left(4.35 \mathrm{~J} \mathrm{~K}^{-1} \mathrm{~mol}^{-1}\right)$, the difference probably arises due the fact that the harmonic approximation applied here does not account for the change in volume with temperature of the phases .

We also investigate which phonon modes are responsible for the difference in $T S_{V i b}$ between the two phases. The frequency dependencies of $T S_{V i b}$ and integrated vibrational entropy $\left(T S_{T o t}\right)$ of the HT and LT structures are plotted in Figs S9a and b, respectively. These plots show how the vibrational modes contribute to the entropy, depending on their frequency. The vast majority of the contribution to $T S_{V i b}$ comes from vibrational modes with frequencies below 10 $\mathrm{THz}$, which leads to the cumulative entropy $\left(T S_{T o t}\right)$ to reach a plateau at around $10 \mathrm{THz}$ at any given temperature, indicating the dominance of low-frequency phonon modes in the lattice vibration. 

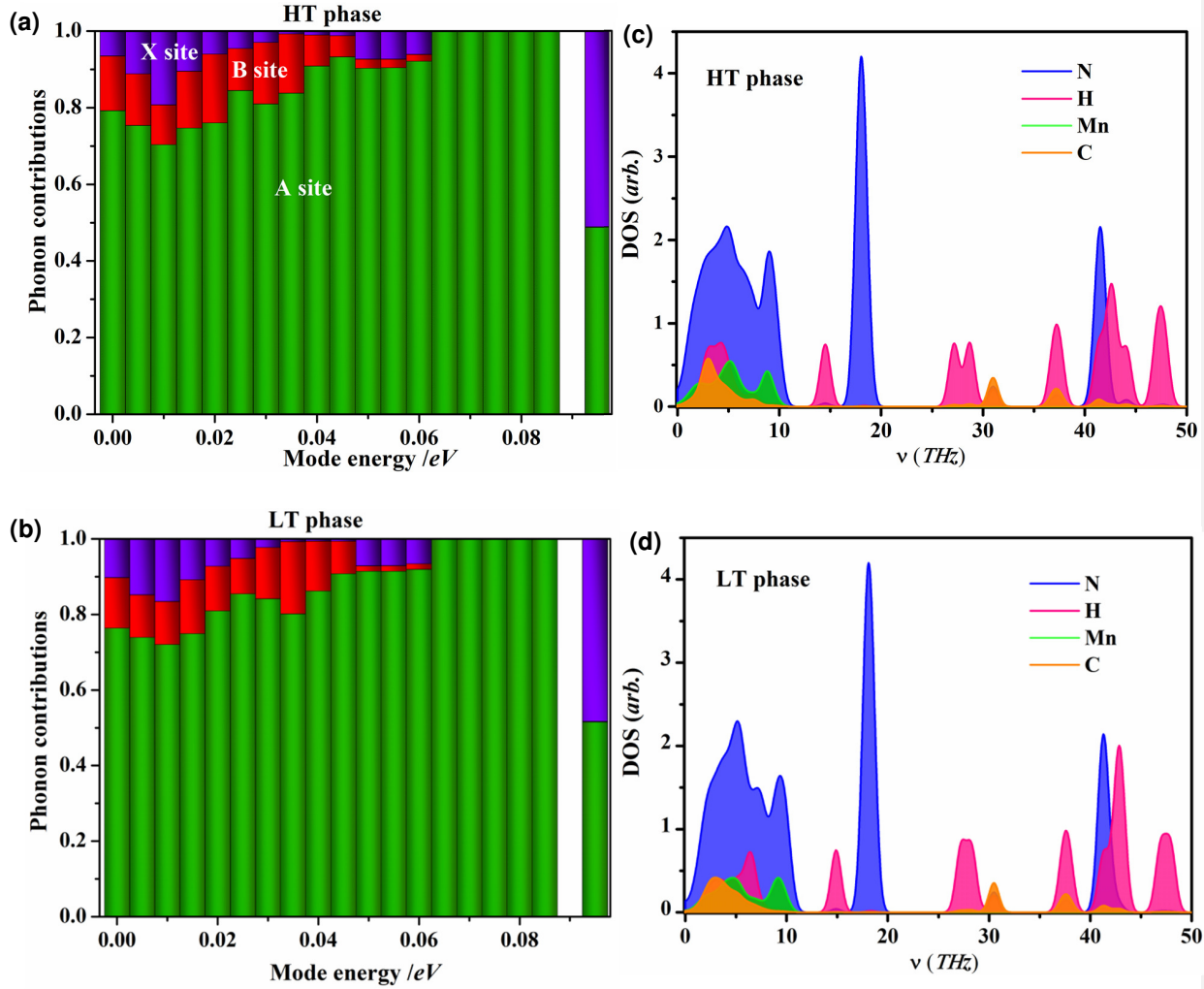

Figure 4. The fractional contribution of A-site (green), B-site (red) and X-site (purple) to the total vibrational entropy at HT (top) (a) and LT (bottom) (b) phase within the $0-0.10 \mathrm{eV}$ energy range. The frequency dependencies of decomposed DOS of each atom at HT (c) and LT phase (d).

To further probe the atomistic origins of the above results, we have decomposed the fractional contribution of each structural site (A-, B- and X-sites) to the $T S_{T o t}$ within the corresponding entropy range by calculating the weighted average of the phonon density of states (DOS) of a given site $i$ at energy $\varepsilon,\left\langle g_{i}(\varepsilon)\right\rangle=\sum_{j} \omega_{i j} / \sum_{k} \omega_{k}$, where $\omega_{i j}$ are the weights of the phonon modes involving the site $i$ at energy $\varepsilon$, and $k$ is the list of all sites (Fig. $4 \mathrm{a}$ and $\mathrm{b})^{20}$. The frequency 
dependencies of decomposed DOS of each atom at HT and LT are plotted in Fig. 4c and d, which shows the contributions to the phonon spectrum broken down by atomic site. As seen from this, the modes that contribute most to the entropy (those below $10 \mathrm{THz}$ ) involve contributions from all A-, B- and X-sites. This confirms that these phonons are collective modes, which depend on the interactions between the sites in the crystal structure. The collective motions determine the entropy and in turn depend on the strength of the bonding between the A-site molecule and the perovskite framework.

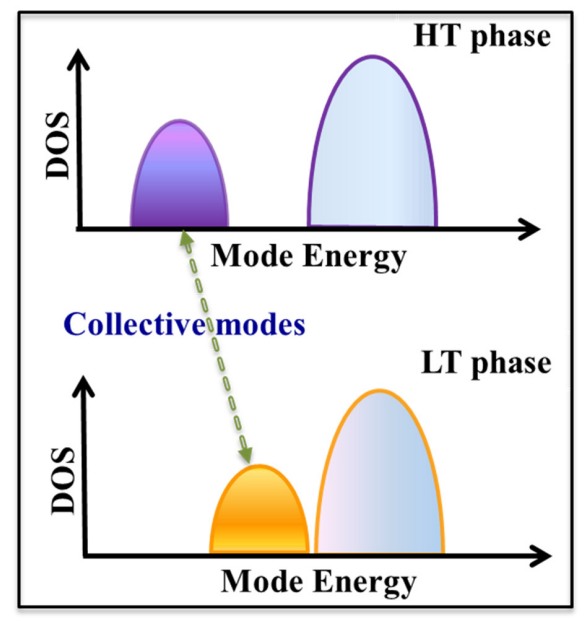

Figure 5. The physical model of the DOS as a function of the mode energy for the HT (top) and LT (bottom) phases influenced by the collective vibrational modes during phase transition. (Keith, what sort of DOS does the high 'hill' mean?)

Based on these findings we can propose a physical model for the forces that drive the PT. In Fig. 5 we show a schematic representation of the HOIP in two different phases. The top phase is the HT phase, which has higher internal energy. The phonon DOS spectra are represented for both phases in the high and low panels, respectively, where the collective modes of the 'softer' HT phase have lower frequency than those of the LT phase. At low temperature the $U$ contribution dominates $G$ and the LT phase is more stable. However, the collective vibrational 
modes become more populated (Boltzmann distribution) with increasing temperature, and the collective modes of the HT phase fill up more rapidly than those of the LT phase so that the vibrational entropy of HT consequently increases at a faster rate. Eventually the balance of free energy between the two phases is reversed as the difference in $T S_{V i b}$ becomes greater than the difference in $U$. This mechanism highlights the important role of collective modes and the delicate balance of inter atomic forces in determining phase stability through accumulative atomic vibrations in certain hybrid materials. It is worth mentioning that such kinds of transitions are significantly different from widely reported order-disorder transitions which feature large entropy changes due to configurational alterations.

In conclusion, we have comprehensively investigated the first order PT in a HOIP, $\left[\mathrm{CH}_{3} \mathrm{NH}_{3}\right]\left[\mathrm{Mn}\left(\mathrm{N}_{3}\right)_{3}\right]$, via a combined approach of experiments and lattice dynamics calculations. The transition is between two different subgroup structures of the parent cubic structure and is representative of the very substantial increase in diversity of structures, properties and PTs which is emerging from this new class of materials in comparison with conventional oxide perovskites. In particular, the difference in vibrations of all atomic sites between the HT and LT phases induces significant energetic changes, hence becoming the main driving force of the transition. Moreover, the large entropic effect, arising solely from vibrational entropy, across the transition is rare in molecular systems since entropy changes normally originate from configurational alterations related to different molecular ordering states. Taking the advantage of abundant chemical diversity of HOIPs, this study unveils a little corner of their giant PT landscape and highlights the fundamentally different new features of these transitions from an atomic level. Furthermore, considering the fact that many important properties of perovskites (e. g. ferroelectricity and multiferroicity) are a consequence of PTs, the cooperative contributions of all A-, B- and X-sites for driving PTs in HOIPs can give rise 
to many novel functionalities that conventional perovskites are unable to endow. Finally, viewing from a broad perspective, we believe this interplay between thermodynamic driving mechanisms - balancing entropy, electrostatics and hydrogen bonding - can stimulate and guide the discovery of new ferroic HOIPs and other hybrid materials.

\section{Methods}

Methods, including statements of data availability and any associated accession codes and references, are available in the online version of this paper.

\section{References}

1. Saparov, B. \& Mitzi, D. B. Organic-inorganic perovskites: structural versatility for functional materials design. Chem. Rev. 116, 4558-4596 (2016).

2. $\mathrm{Li}, \mathrm{W}$. et al. Chemically diverse and multifunctional hybrid organic-inorganic perovskites. Nat. Rev. Mater. 16099 (2017).

3. Xu, W.-J., Du, Z.-Y. \& Zhang, W.-X. et al. Structural phase transitions in perovskite compounds based on diatomic or multiatomic bridges[J]. CrystEngComm 18, 7915 (2016).

4. Whitfield, P. S. et al. Structures, Phase Transitions and Tricritical Behavior of the Hybrid Perovskite Methyl Ammonium Lead Iodide. Sci. Rep. 6, 35685 (2016).

5. Howard, C. J. \& Stokes, H. T. Group-theoretical analysis of octahedral tilting in perovskites. Acta Crystallogr. B 54, 782-789 (1998).

6. Stokes, H. T., Kisi, E. H., Hatch, D. M. \& Howard, C. J. Group-theoretical analysis of octahedral tilting in ferroelectric perovskites. Acta Crystallogr. B 58, 934-938 (2002).

7. Boström, H. L. B., Hill, J. A. \& Goodwin, A. L. Columnar shifts as symmetry-breaking degrees of freedom in molecular perovskites[J]. Phys. Chem. Chem. Phys. 18(46): 31881-31894 (2016).

8. Gómez-Aguirre, L. C. et al. Coexistence of three ferroic orders in the multiferroic compound $\left[\left(\mathrm{CH}_{3}\right)_{4} \mathrm{~N}\right]\left[\mathrm{Mn}\left(\mathrm{N}_{3}\right)_{3}\right]$ with perovskite-like structure. Chem. Eur. J. 22, 1-9 (2016).

9. Du, Z. Y., Sun, Y. Z., Chen, S. L. et al. Insight into the molecular dynamics of guest cations confined in deformable azido coordination frameworks[J]. Chem. Commun. 51(86): 15641-15644 (2015).

10. Du, Z. Y., Xu, T. T., Huang, B. et al. Switchable Guest Molecular Dynamics in a Perovskite-Like Coordination Polymer toward Sensitive Thermoresponsive Dielectric Materials[J]. Angew. Chem. Int. Ed. 54(3): 914-918 (2015).

11. Jain, P., Dalal, N. S., Toby, B. H. et al. Order-disorder antiferroelectric phase transition in a hybrid inorganic-organic framework with the perovskite architecture[J]. J. Am. Chem. Soc. 130(32): 10450-10451 (2008).

12. Zhang, W., Cai, Y., Xiong, R. G. et al. Exceptional Dielectric Phase Transitions in a Perovskite-Type Cage Compound[J]. Angew. Chem. 122(37): 6758-6760 (2010).

13. Bermúdez-García J M, Sánchez-Andújar M, Yáñez-Vilar S, et al. Multiple phase and dielectric transitions on a novel multi-sensitive $[\mathrm{TPrA}]\left[\mathrm{M}(\mathrm{dca})_{3}\right]\left(\mathrm{M}: \mathrm{Fe}^{2+}, \mathrm{Co}^{2+}\right.$ and 
$\mathrm{Ni}^{2+}$ ) hybrid inorganic-organic perovskite family[J]. J. Mater. Chem. C 4(22): 48894898 (2016).

14. Mitzi, D. B. Synthesis, structure, and properties of organic-inorganic perovskites and related materials. Prog. Inorg. Chem. 48, 1-121 (1999).

15. Shang, R., Chen, S., Wang, B., Wang, Z. \& Gao, S. Temperature-induced irreversible phase transition from perovskite to diamond but pressure-driven backtransition in an ammonium copper formate. Angew. Chem. Int. Ed. 55, 2097-2100 (2016).

16. Hill, J. A., Thompson, A. L. \& Goodwin, A. L. Dicyanometallates as model extended frameworks. J. Am. Chem. Soc. 138, 5886-5896 (2016).

17. Fu, D. et al. A multiferroic perdeutero metal-organic framework. Angew. Chem. Int. Ed. 50, 11947-11951 (2011).

18. Zhang, W. \& Xiong, R. Ferroelectric metal-organic frameworks. Chem. Rev. 112 1163-1195 (2012).

19. Hang, T., Zhang, W., Ye, H. \& Xiong, R. Metal-organic complex ferroelectrics. Chem. Soc. Rev. 40, 3577-3598 (2011).

20. Kieslich, G. et al. Role of entropic effects in controlling the polymorphism in formate $\mathrm{ABX}_{3}$ metal-organic frameworks. Chem. Commun. 51, 15538-15541 (2015).

21. Zhao, X. et al. Cation-dependent magnetic ordering and room-temperature bistability in azido-bridged perovskite-type compounds. J. Am. Chem. Soc. 135, 16006-16009 (2013).

22. Howard, C. J. \& Stokes, H. T. Group-theoretical analysis of octahedral tilting in perovskites. Acta Crystallogr. B 54, 782-789 (1998).

23. Stokes, H. T., Hatch, D. M., Campbell, B. J. 2007. ISOTROPY. $<\mathrm{http}: / /$ www.stokes.byu.edu/isotropy.html $>$.

24. Carpenter, M. A. Static and dynamic strain coupling behaviour of ferroic and multiferroic perovskites from resonant ultrasound spectroscopy. J. Phys.: Condens. Matter. 27, 263201 (2015).

25. Stokes, H. T., Orden, S., Campbell, B. J. ISOSUBGROUP: an internet tool for generating isotropy subgroups of crystallographic space groups[J]. J. Appl. Crystallogr. 49(5):, 1849-1853 ( 2016).

26. Migilori, A., Sarraro, J. L. Resonant ultrasound spectroscopy: applications to physics, material measurements and non-destructive evaluation. New York: Wiley; 1997.

27. McKnight, R. E. A., Carpenter, M. A., Darling, T. W. et al. Acoustic dissipation associated with phase transitions in lawsonite, $\mathrm{CaAl}_{2} \mathrm{Si}_{2} \mathrm{O}_{7}(\mathrm{OH})_{2} \cdot \mathrm{H}_{2} \mathrm{O}$. Am. Mineral. 92, 1665-1672 (2007).

28. Li, W. et al. Ferroelasticity in a metal-organic framework perovskite; towards a new class of multiferroics. Acta Materialia 61, 4928-4938 (2013).

29. Thomson, R. I., Jain, P., Cheetham, A. K. \& Carpenter, M. A. Elastic relaxation behavior, magnetoelastic coupling, and order-disorder processes in multiferroic metalorganic frameworks. Phys. Rev. B 86, 214304 (2012).

30. Butler, K. T., Svane, K, Kieslich, G. et al. Microscopic origin of entropy-driven polymorphism in hybrid organic-inorganic perovskite materials[J]. Phys. Rev. B 94(18): 180103 (2016).

31. Butler, K.T., Walsh, A., Cheetham, A. K. \& Kieslich, G. Organised chaos: entropy in hybrid inorganic-organic systems and other materials. Chem. Sci. 7, 6316-6324 (2016).

32. Stoumpos, C. C., Malliakas, C. D. \& Kanatzidis, M. G. Semiconducting tin and lead iodide perovskites with organic cations: phase transitions, high mobilities, and nearinfrared photoluminescent properties. Inorg. Chem. 52, 9019-9038 (2013). 
33. Jain, P. et al. Multiferroic behavior associated with an order-disorder hydrogen bonding transition in metal-organic frameworks (MOFs) with the perovskite $\mathrm{ABX}_{3}$ architecture. J. Am. Chem. Soc. 131, 13625-13627 (2009).

\section{Acknowledgements}

We acknowledge financial support from NSFC (Grant Nos. 21571072, 11374114 and 10974062).

\section{Author contributions}

W.L. designed and organised the research. Experiments were performed by W.W. and W.L. Theoretical calculations were performed by K.T.B. Data analysis was performed by W.W., W.L., K.T.B., A.W., M.A.C. and C.J.H. W.W., W.L., C.J.H. and K.T.B. wrote the full manuscript, and A.W., M.A.C., S.G., G.F. and P.L. contributed to correcting the paper.

\section{Additional information}

The authors declare no competing financial interests. Supplementary information accompanies this paper at www.nature.com/naturechemistry. Reprints and permission information is available online at http://npg.nature.com/reprintsandpermissions/. Correspondence and requests for calculations should be addressed to W.L., K.T.B., S.G. or M.A.C. 